\title{
Does modulation of selective attention to features reflect enhancement or suppression of neural activity?
}

\section{Citation}

Daffner, Kirk R., Tatyana Y. Zhuravleva, Xue Sun, Elise C. Tarbi, Anna E. Haring, Dorene M. Rentz, and Phillip J. Holcomb. 2012. "Does Modulation of Selective Attention to Features Reflect Enhancement or Suppression of Neural Activity?" Biological Psychology 89 (2) (February): 398407. doi:10.1016/j.biopsycho.2011.12.002.

\section{Published Version}

doi:10.1016/j.biopsycho.2011.12.002

\section{Permanent link}

http://nrs.harvard.edu/urn-3:HUL.InstRepos:32604929

\section{Terms of Use}

This article was downloaded from Harvard University's DASH repository, and is made available under the terms and conditions applicable to Other Posted Material, as set forth at http:// nrs.harvard.edu/urn-3:HUL.InstRepos:dash.current.terms-of-use\#LAA

\section{Share Your Story}

The Harvard community has made this article openly available.

Please share how this access benefits you. Submit a story.

Accessibility 


\title{
Does modulation of selective attention to features reflect enhancement or suppression of neural activity?
}

\author{
Kirk R. Daffner ${ }^{1}$, Tatyana Y. Zhuravleva ${ }^{1}$, Xue Sun ${ }^{1}$, Elise C. Tarbi ${ }^{1}$, Anna E. Haring ${ }^{1}$, \\ Dorene M. Rentz ${ }^{1}$, and Phillip J. Holcomb ${ }^{2}$ \\ ${ }^{1}$ Center for Brain/Mind Medicine, Division of Cognitive and Behavioral Neurology, Department of \\ Neurology, Brigham and Women's Hospital, Harvard Medical School, 221 Longwood Avenue, \\ Boston, MA 02115 \\ ${ }^{2}$ Department of Psychology, Tufts University, 490 Boston Avenue, Medford, MA 02155
}

\begin{abstract}
Numerous studies have demonstrated that selective attention to color is associated with a larger neural response under attend than ignore conditions, but have not addressed whether this difference reflects enhanced activity under attend or suppressed activity under ignore. In this study, a color-neutral condition was included, which presented stimuli physically identical to those under attend and ignore conditions, but in which color was not task relevant. Attention to color did not modulate the early sensory-evoked P1 and N1 components. Traditional ERP markers of early selection (the anterior Selection Positivity and posterior Selection Negativity) did not differ between the attend and neutral conditions, arguing against a mechanism of enhanced activity. However, there were markedly reduced responses under the ignore relative to the neutral condition, consistent with the view that early selection mechanisms reflect suppression of neural activity under the ignore condition.
\end{abstract}

\section{Introduction}

Although many studies have shown that selective attention to color is associated with a larger neural response under attend than ignore conditions, they have not addressed whether this difference reflects enhanced activity under attend or suppressed activity under ignore. Understanding this process is critical to determining the ways in which top-down modulation of information processing is carried out, and delineating the mechanisms that may underlie attentional impairment in clinical populations.

Selective visual attention reflects the influence of top-down control mechanisms that facilitate the processing of information most relevant to task demands (Desimone and Duncan, 1995; Lavie et al., 2004; Rutman et al., 2010; Sawaki and Katayama, 2008). As a result of these operations, selective attention is hypothesized to improve processing efficiency and conserve resources of the capacity-limited decision making system (Awh and Jonides, 2001; Gazzaley et al., 2005a; Zanto and Gazzaley, 2009). Selective attention has

(C) 2011 Elsevier B.V. All rights reserved.

*Corresponding author: Kirk R. Daffner, M.D., Division of Cognitive and Behavioral Neurology, Brigham and Women's Hospital, Harvard Medical School, 221 Longwood Avenue, Boston, MA 02115, U.S.A. (617) 732-8060 (phone); (617) 738-9122 (fax); kdaffner@partners.org.

Publisher's Disclaimer: This is a PDF file of an unedited manuscript that has been accepted for publication. As a service to our customers we are providing this early version of the manuscript. The manuscript will undergo copyediting, typesetting, and review of the resulting proof before it is published in its final citable form. Please note that during the production process errors may be discovered which could affect the content, and all legal disclaimers that apply to the journal pertain. 
been investigated using event-related potential (ERP) and functional imaging techniques. The most common ERP investigations of selective attention have involved attention to location and reveal an amplification (increased gain) of early sensory-evoked components such as the $\mathrm{P} 1$ or $\mathrm{N} 1$, which results in a change in the size of the component, but not its latency, morphology, or scalp distribution (Hillyard et al., 1998b).

In contrast to attending to location, selective attention to non-spatial features such as color has most often been associated with the generation of endogenous potentials and not the modulation of sensory-evoked ERP components (Hillyard and Anllo-Vento, 1998; Hillyard and Munte, 1984). In standard investigations of attention to color, comparing attend and ignore conditions, two ERP modulations are most commonly described: a Selection Negativity (SN) over posterior scalp locations and a Selection Positivity (SP) over frontocentral regions (Czigler, 1996; Eimer, 1997; Hillyard et al., 1998a; Kopp et al., 2007; Luck and Hillyard, 1994; Martin-Loeches et al., 1999; Potts and Tucker, 2001; Van Der Stelt et al., 1998). These potentials exhibit an overlapping time course between $~ 150-350$ $\mathrm{ms}$ post stimulus presentation. There is evidence that the posterior SN reflects the activity of feature-selection areas of the extrastriate cortex that may involve the enhanced sensoryperceptual processing of relevant stimulus dimensions compared to irrelevant ones (Harter and Aine, 1984; Hillyard et al., 1998a; Kopp et al., 2007). The neural underpinnings and functional significance of the anterior SP are less well established. It has been conceptualized either as a frontally mediated index of the motivational salience of a stimulus based on task relevance (Potts and Tucker, 2001; Riis et al., 2009) or as a marker of a detection process sensitive to stimulus features, such as color, orientation, or size that have been specified by task instructions as being significant (Luck and Hillyard, 1994).

A few recent studies have also reported modulations of early sensory-evoked potentials in response to attention to color. For example, Zhang and Luck (2009) found enhanced P1 amplitude to an attended relative to an ignored color at an unattended location, but only under conditions in which attended and ignored colors were presented simultaneously rather than sequentially. Zanto and colleagues (2010b) found a larger N1 amplitude to colored stimuli under an attend than ignore condition when they showed subjects a series of colored or moving dots, and compared the effects of attending to vs. ignoring the feature of color.

Most commonly, the study of selective attention has involved a comparison of the neural activity elicited by the same physical stimuli that are presented under attend vs. ignore conditions, often by using subtraction techniques (attend minus ignore). The standard subtraction method is able to demonstrate that a difference exists between attending and ignoring. However, it cannot determine whether the difference reflects increased activity under the attend condition, reduced activity under the ignore condition, or both. One rarely utilized strategy to address this challenge has been to include a condition that is attentionally neutral with respect to the feature under consideration. For example, Luck et al. (1994) tested subjects in a spatial cueing experiment that included "neutral" trials in which the cues pointed toward all possible locations, presumably leading subjects not to focus spatial attention. Comparing valid to neutral trials provided an index of attentional enhancement, whereas comparing invalid to neutral trials provided an index of attentional suppression. Interestingly, the amplitude of the sensory-evoked P1 component was smaller on invalid (ignore) than neutral trials, with no enhancement on valid (attend) trials. In contrast, the N1 component only showed enhancement on valid (attend) relative to neutral trials, but no suppression on invalid (ignore) relative to neutral trials.

A similar approach has been applied to the study of non-spatial features. Gazzaley and colleagues (2005a; 2005b; 2008; Zanto et al., 2010a) have used a neutral condition to investigate selective attention in several functional imaging and ERP experiments. For 
example, subjects were shown a series of faces and scenes under three conditions: 1) remember faces (and ignore scenes), 2) ignore faces (and remember scenes), and 3) passively view both faces and scenes. In one of the ERP experiments, young adults exhibited both significant enhancement (attend > passive view) and suppression (passive view > ignore) for P1 amplitude and N1 latency in response to faces (Gazzaley et al., 2008). ERP studies of selective attention to color that have used a neutral condition have been extremely rare. In the Zanto et al. study (2010b) cited earlier, subjects viewed a sequence of colored or moving dots under four experimental conditions and were instructed to attend to motion, hue, both motion and hue, or to just passively view the stimuli. Subjects demonstrated enhancement (attend $>$ passive view) but not suppression (passive view $=$ ignore) of the N1 amplitude in response to color. Although Selection Negativity was examined (attend - ignore difference waves), the authors did not compare activity under attend or ignore conditions with activity under the passive condition.

In the current study, attention to a specified color was investigated by comparing attend and ignore conditions to a neutral condition. Young adult subjects were shown a series of red and blue letters, of which five were designated as targets. Under the color-selective attention task, subjects were told to respond to target letters in a designated color and to ignore stimuli in the other color. Under the color-neutral attention task subjects were told to respond to target letters that appeared in either color. The color-selective attention (SA) task allowed us to examine the neural responses to stimuli in which subjects were instructed to attend (Attend condition) or to ignore (Ignore condition) based on color. The color-neutral attention (NA) task allowed us to examine the neural responses to physically identical stimuli under circumstances in which color was not task-relevant (Neutral condition). The critical difference between the SA task and the NA task is the additional requirement of color selectivity. Our working assumption was that carrying out the SA task included all of the cognitive operations involved in the NA task, plus ones that mediate the color selection process. For example, both tasks required subjects to actively attend to the experiment, focus gaze on a central location, perceptually process the physical stimuli, make decisions about whether the presented stimulus matches one of the target letters, prepare and execute the appropriate motor responses, update memory, and prepare for the next stimuli. However, under the SA task, subjects had to account for color as well as letter forms.

Selection of color and letter forms may be hierarchical (with the processing of one dimension dependent on the outcome of prior selection within the other dimension), or may take place in parallel. Theories about early selection lead to the expectation that subjects would initially filter input on the basis of the most easily identifiable physical characteristic (color) linked to target stimuli, and then process more complex features (letter forms) of stimuli in the relevant color in order to complete target identification activity (Hillyard and Munte, 1984; Looren et al., 1988). A potential challenge to this version of a hierarchical framework is the observation that in experienced readers, words are automatically (and rapidly) processed, which, for example, manifests in the Stroop effect (MacLeod, 1991). In accordance with this idea, the letter forms presented in the current study would be processed first, target letters identified, and then color attributes determined in order to complete the selection of appropriate targets. In this scenario, discrimination of color would reflect a late selection process, occurring in the service of final target identification. Against this possibility are studies indicating that the Stroop effect is attenuated or absent using a single letter format (Besner et al., 1997; Brown et al., 2002). Functional imaging studies have also found that single letters do not reliably engage the fusiform visual word form area (Turkeltaub et al., 2008). In ERP studies, the N170 component has been used as an index of the processing of word forms. The N170 sensitive to word forms is larger over left hemisphere sites (Maurer et al., 2008; Rossion et al., 2003). In the current study, if subjects treated individual letters like whole word forms and thus processed them in a rapid, 
automatic fashion, we would expect to find a larger N170 component over the left hemisphere.

An alternative to these hierarchical models of selection would suggest that the attributes of color and letter form are processed in parallel by independent analyzers. Theoretically, even in this model, once stimuli are identified as lacking a critical attribute, processing of that stimulus could be terminated (Hillyard and Munte, 1984). Letter forms represent a less discriminable feature than color, and likely require more time to resolve. Consistent with this framework, color selection would take place early, and play an important role in efficiently carrying out the task demands of identifying targets.

Analysis of ERPs is particularly well-suited for determining where along the information processing stream color selection takes place. We examined the posterior P1, posterior N1 (N170), SP, and SN components. Based on the extant literature, we expected that the most salient changes would be observed during the temporal intervals traditionally associated with color processing, the anterior SP and posterior SN. The ERP subtraction methodology allowed us to focus on color processing. For example, the comparison of color positive, letter form negative $(\mathrm{C}+/ \mathrm{L}-)$ events to color negative, letter-form negative $(\mathrm{C}-/ \mathrm{L}-)$ events is a way to isolate color selection by subtracting out processing activity specifically related to letter forms.

We hypothesized that under the SA task, selective attention to specific features of a stimulus (e.g., color) would modulate operations elicited under the NA task and/or elicit additional ones. For example, if the size of a component were larger under the Attend condition than under the Neutral and Ignore conditions, with no difference between the latter two (represented as A > N = I), it would suggest that the difference between the Attend and Ignore conditions is explained by the enhanced activity involved with selection of specific features (color), which is not required under the Neutral condition $(A>N)$. There would be no evidence to suggest a reduction of activity under the Ignore relative to Neutral conditions, as both conditions elicit a similar level of activity $(\mathrm{N}=\mathrm{I})$. By contrast, if the size of the response was larger under the Attend and Neutral conditions than the Ignore condition, with no difference between the former two (represented as $\mathrm{A}=\mathrm{N}>\mathrm{I}$ ), it would suggest that there was no increase of neural activity associated with the selective attention operations beyond those involved with the more general process of directing attention to stimuli $(A=N)$. This pattern would also indicate that there was less neural activity under the Ignore condition than under the Neutral condition. In keeping with other studies (Gazzaley et al., 2005a; Gazzaley et al., 2005b), we interpret the N > I pattern as representing suppression of activity under the Ignore condition.

\section{Materials and Methods}

\section{Participants}

Twenty five healthy subjects between the ages of 18-32 ( 13 females; mean age $22.6 \pm 2.3$; mean education $15.1 \pm 1.6$ years; mean estimated IQ $116.6 \pm 6.8$ based on the AMNART (Ryan and Paolo, 1992)) participated in this study. Three additional subjects were excluded due to excessively noisy data. All participants were free of CNS diseases or major psychiatric disorders based on DSM-IV criteria (American Psychiatric Association, 1994), had a Beck Depression Inventory (Beck and Steer, 1987) score of < 10, were able to distinguish between the color red and blue, and had a corrected visual acuity of at least 2040 as tested by a Snellen wall chart. Written informed consent was obtained from all subjects before the study. Subjects were paid for their time. 


\section{Experimental Methods}

The experiment consisted of two related tasks: a color-selective attention (SA) task from which the Attend (A) and Ignore (I) conditions were extracted, and a color-neutral attention (NA) task from which the Neutral (N) condition was derived. In both tasks, subjects were shown physically identical sets of stimuli, which consisted of a series of letters presented in either the color red or the color blue, and were asked to respond by button press to 5 specific target letters. In the SA task, subjects were instructed to pay attention to letters appearing in the designated color while ignoring letters appearing in the other color, and to respond by button press to target letters appearing in the designated color only. In the NA task, subjects were instructed to pay attention to all letters regardless of color. Under both tasks, subjects were asked to respond as quickly and as accurately as possible to target letters. Practice trials preceded each set of experimental trials. All subjects participated in both tasks, the order of which was counterbalanced. To reduce potential order effects, each task was presented during a different experimental session, separated by at least 2 weeks. The hand used for the target response was counterbalanced across subjects, as was the attended color in the SA condition. Additionally, the 5 specific letters selected as targets differed between the NA and the SA tasks.

Each task included 800 stimulus trials divided into 8 blocks. Under both tasks, stimuli appeared one at a time within a fixation box that remained on the screen at all times and subtended a visual angle of $\sim 3.5^{\circ} \times 3.5^{\circ}$ at the center of a high-resolution computer monitor. Half of the stimuli appeared in the color red and half in the color blue, in randomized order. ${ }^{1}$ Target stimuli (SA: 7.5\% in attend color; 7.5\% in ignore color; NA: 7.5\% probability overall, $3.75 \%$ in each color) were 5 designated upper case letters and standard stimuli (SA: $70 \%$ overall; $35 \%$ in each color; NA: $77.5 \%$ overall, $38.75 \%$ in each color) were any nontarget upper case letters. ${ }^{2}$ Fillers accounted for the remainder of the stimuli presented.

Visual stimuli subtended an angle of $2.5^{\circ}$ along their longest dimension and were presented for $250 \mathrm{~ms}$. The inter-stimulus interval (ISI) varied randomly between $815-1015 \mathrm{~ms}$ (mean $\sim 915 \mathrm{~ms}$ ) (see Fig. 1). For analytic purposes, trials under the SA task were further categorized in terms of whether the stimuli presented were in the attend or the ignore color. The Attend condition consisted of all stimuli in the designated color; the Ignore condition consisted of all stimuli in the non-designated color.

\section{ERP Recordings}

An ActiveTwo electrode cap (Behavioral Brain Sciences Center, Birmingham, UK) was used to hold to the scalp a full array of $128 \mathrm{Ag}-\mathrm{AgCl}$ BioSemi (Amsterdam, The Netherlands) "active" electrodes whose locations were based on a pre-configured montage. Electrodes were arranged in equidistant concentric circles from 10-20 position Cz. In addition to the 128 electrodes on the scalp, 6 mini bio-potential electrodes were placed over the left and right mastoid, beneath each eye, and next to the outer canthi of the eyes to check for eye blinks and vertical and horizontal eye movements. EEG activity was digitized at a sampling rate of $512 \mathrm{~Hz}$.

\footnotetext{
${ }^{1}$ Under the SA task, the task-relevant color remained the same throughout the experiment (which has been labeled sustained attention to color) rather than having the pertinent color cued before each trial (which has been labeled transient attention to color). This experimental design was chosen because ERP differences between attend and ignore conditions (as indexed by the SP and SN components) have been shown to be much larger under tasks that require sustained rather than transient attention to color (Eimer, 1997). Note that this approach differs from the studies of selective attention and working memory conducted by Gazzaley et al. (2005a; 2005b; 2008; Zanto et al., 2010a) and Zanto et al. (2010b; Zanto and Gazzaley, 2009), in which the stimulus set changed on each trial.

${ }^{2}$ The percentage of stimuli designated as targets was held constant across all conditions to encompass $7.5 \%$ of total stimuli. Since subjects were to respond to target letters in only one color under the SA task but to respond to target letters in both colors under the NA task, the overall probability of encountering a target letter under the NA task was reduced by half to $7.5 \%$ (3.75\% in each color). More standards were added to accommodate for this difference.
} 


\section{Data Analysis}

Mean reaction time (RT) and accuracy rates were measured separately in the SA and NA tasks. A response was considered a hit if it occurred between $200-1000 \mathrm{~ms}$ after stimulus presentation. Target stimuli correctly responded to (Target Hits) and stimuli incorrectly identified as targets (False Alarms) were measured in order to determine an overall accuracy score (Percent Target Hits- Percent False Alarms). Performance under the 2 tasks was compared using paired t-tests.

EEG data were analyzed using ERPLAB (www.erpinfo.org/erplab) and EEGLAB (Delorme and Makeig, 2004; http://sccn.ucsd.edu/eeglab) toolboxes that operate within the MATLAB framework. Raw EEG data were resampled to $256 \mathrm{~Hz}$ and referenced off-line to the algebraic average of the right and left mastoids. EEG signals were filtered using an IIR filter with a bandwidth of $0.03-40 \mathrm{~Hz}$ ( $12 \mathrm{~dB} /$ octave roll-off). Eye artifacts were removed through an independent component analysis. Individual bad channels were corrected with the EEGLAB interpolation function. EEG epochs for the two stimulus types (standard stimuli, target hits/target-like stimuli) across three attention conditions (Attend, Ignore, Neutral) were averaged separately. The sampling epoch for each trial lasted for $1200 \mathrm{~ms}$, including a $200 \mathrm{~ms}$ pre-stimulus period that was used to baseline correct the ERP epochs. Trials were discarded from the analyses if they contained baseline drift or movement artifacts greater than $90 \mu \mathrm{V}$. Only trials with correct responses were included in the analyses.

Regions of Interest (ROIs) across the scalp were designated and labeled Centro-Frontal (CF), Left Anterior Lateral (LAL), Right Anterior Lateral (RAL), Left Anterior Medial (LAM), Right Anterior Medial (RAM), Left Posterior Medial (LPM), Right Posterior Medial (RPM), Left Occipito-Temporal (LOT), Right Occipito-Temporal (ROT), and Centro-Occipital (CO) (see Fig. 2). Each region reflected a cluster of 7 electrode sites. Only ERPs to standard stimuli were analyzed here to avoid the potential confounding influence of motor components associated with responses to target stimuli under the Attend and Neutral conditions, but not the Ignore condition. The latency of the posterior P1 was measured as the local positive peak latency under the Attend, Ignore, and Neutral conditions between 50-150 $\mathrm{ms}$ at the LOT and ROT ROIs. The latency of the posterior N1 was measured as the local negative peak latency under the Attend, Ignore, and Neutral conditions between 125-225 ms at the LOT and ROT ROIs. The size of the P1 and N1 components was measured as the mean amplitude of the $20 \mathrm{~ms}$ interval centered at the mean peak latency for each component. The latency of the SP was measured as the local positive peak latency for the Attend- Ignore and Neutral- Ignore difference waves ${ }^{3}$ between 100-275 ms at anterior ROIs. The latency of the SN was measured as the local negative peak latency for the Attend - Ignore and the Neutral - Ignore difference waves ${ }^{3}$ between $200-350 \mathrm{~ms}$ in response to standard stimuli at posterior ROIs. The size of the SP and SN was derived from the mean amplitude of the 100 ms interval centered at the mean peak latency for each component. ERPs were analyzed using analysis of variance (ANOVA), with attention condition (Attend, Ignore, Neutral) and ROI as within-subject variables. The Geisser-Greenhouse correction was applied for all repeated measures with greater than 1 degree of freedom. Of note, preliminary ANOVAs revealed that the size of the $\mathrm{P} 1, \mathrm{~N} 1, \mathrm{SP}$, and $\mathrm{SN}$ did not differ as a function of which color was attended. Thus, ERPs were combined across subjects who had been instructed to attend to the red or blue color.

${ }^{3}$ For the SP, the local positive peak latency for the Attend - Neutral difference wave was not measured because there were no consistent differences in amplitude between the Attend and Neutral conditions. For the SN, the local negative peak latency for the Attend - Neutral difference wave was not measured for the same reason. 


\section{Results}

\section{Behavior}

Mean RTs were slower under the SA task (605.8 (55.0) ms) than the NA task (567.4 (66.4) $\mathrm{ms})\left(\mathrm{t}_{24}=4.62, \mathrm{p}<0.00001\right)$. However, accuracy did not differ between conditions.

\section{ERPs}

The grand average ERP responses to standard stimuli under the Attend, Ignore, and Neutral conditions at all 10 ROIs are presented in Figure 3. Representative ROIs are featured in subsequent figures, as described below.

Posterior P1 Component-The mean local positive peak latency of the posterior P1 at ROIs LOT and ROT was 102.2 (3.0) ms. There was no effect of condition or ROI. The amplitude of the P1 was thus measured as the mean value between $92-112 \mathrm{~ms}$ in response to standard stimuli at ROIs LOT and ROT (Fig. 4b). There was no effect of condition (p > $0.42)$. There was an effect of ROI $\left(F_{1,23}=9.92, p<0.005\right)$, which was present because the $\mathrm{P} 1$ amplitude was larger over right than left occipital-temporal sites. The magnitude of this effect was similar across conditions (no ROI x condition interaction).

Posterior N1 (N170) Component-The mean local peak negative latency of the posterior N1 at ROIs LOT and ROT was 162.0 (2.0) ms. There was no effect of condition or ROI. The amplitude of the $\mathrm{N} 1$ was thus measured as the mean value between $152-172 \mathrm{~ms}$ in response to standard stimuli at ROIs LOT and ROT (Fig. 4b). There was no effect of condition ( $\mathrm{p}>0.17)$. Of particular interest for determining whether the letter stimuli generated a word N170, the size of this component was not larger over left hemisphere sites (no effect of ROI, $\mathrm{p}>0.98$ ).

Anterior Selection Positivity-Figure 4a highlights the grand average ERPs at the anterior ROI, CF. Figure 5a presents the difference waves at ROI CF and Figure 5b presents the surface potential maps of the Attend - Ignore, Neutral - Ignore, and Attend - Neutral difference waves during the temporal interval of the SP, which illustrate that the most salient differences in the Selection Positivity between conditions occurred at anterior scalp regions. Of note, both the A-I and the N-I difference waves demonstrate large positive deflections in the temporal window measured (Fig. 5a). In contrast, the A-N difference wave has a biphasic appearance, with an initial negative deflection $(N>A)$, followed by a positive deflection $(\mathrm{A}>\mathrm{N})$. The reason for this pattern can be discerned from the grand average plots (Fig. 4a). Initially, the response under the Neutral condition is larger than under the Attend condition. However, during the latter portion of the interval, the positivity under the Attend condition is larger than under the Neutral condition. 4

The mean positive peak latency of the SP difference waves was 180.8 (3.6) ms. Although the SP value appeared to peak later for the A-I than the N-I difference waves, the difference in latency was not reliable ( $p>0.1$ ). The amplitude of the Anterior Selection Positivity (SP) was thus measured as the mean value between $130-230 \mathrm{~ms}$ at the 5 anterior ROIs (CF, LAL, RAL, LAM, RAM) (Fig. 3). An ANOVA revealed an effect of condition $\left(\mathrm{F}_{2,48}=8.99, \mathrm{p}<\right.$ 0.004 ) that did not differ across ROIs (no condition $x$ ROI interaction). The condition effect was due to the mean amplitude being more positive under both the Attend condition and the

\footnotetext{
${ }^{4}$ An initial review of the difference waves may lead to the impression that the N-I difference wave is also biphasic, with both the negative and positive deflections having an earlier latency than the A-N difference wave. However, closer inspection reveals that the negative deflection between $75-100 \mathrm{~ms}$ in the N-I difference wave is related to the preceding anterior N1 component, where the Neutral condition is more negative than either the Attend or Ignore conditions (see Fig. 4a), and not the anterior P2 component from which the SP is derived.
} 
Neutral condition than under the Ignore condition, with no difference between the Attend and Neutral conditions ( $\mathrm{A}=\mathrm{N}>\mathrm{I}$; Attend equal to Neutral $(\mathrm{p}>0.24)$; Attend more positive than Ignore $(\mathrm{p}<0.00001)$; Neutral more positive than Ignore $(\mathrm{p}<0.006))$.

To further validate the findings, we also measured the local peak amplitude of the anterior P2, whose modulation results in the anterior Selection Positivity (Hillyard and Munte, 1984; Kopp et al., 2007; Luck and Hillyard, 1994). The results of the ANOVA of the local peak P2 amplitude between 100-275 ms at anterior ROIs followed the same pattern that was observed for the analysis of mean amplitudes. There was an effect of condition $\left(\mathrm{F}_{2,48}=7.38\right.$, $\mathrm{p}<0.006 ; \mathrm{A}=\mathrm{N}>\mathrm{I}$; Attend equal to Neutral ( $>0.83)$; Attend more positive than Ignore $(p<0.00001)$; Neutral more positive than Ignore $(p<0.003)$ ). The magnitude of this effect did not differ across ROIs (no condition x ROI interaction). Of note, after the peak of the P2 component, the positivity under the Attend condition appears to be sustained longer than under the Neutral condition (Fig. 4a). This observation was confirmed by examining the mean amplitude between 200-230 ms, where the value was largest under the Attend condition, with no difference between the Neutral and Ignore conditions (effect of condition, $\left.\mathrm{F}_{2,48}=6.72, \mathrm{p}<0.006\right)$.

Posterior Selection Negativity-Figure 4b highlights the grand average ERPs at the left occipito-temporal (LOT) ROI. Figure 6a presents the difference waves at LOT and Figure $6 \mathrm{~b}$ presents the surface potential maps of the Attend - Ignore, Neutral - Ignore, and Attend Neutral difference waves during the temporal interval of the $\mathrm{SN}$, illustrating that the most prominent differences between conditions occurred at lateral posterior scalp regions. The mean negative peak latency of the SN difference waves at posterior ROIs was earlier for the A-I difference than the N-I difference wave (268.4 (4.7) vs. 284.6 (5.5) $\mathrm{ms}, \mathrm{F}_{1,24}=14.07$, p $<0.001$. The posterior Selection Negativity was measured as the mean amplitude between 225-325 ms in response to standard stimuli at the 5 posterior ROIs (LOT, ROT, LPM, RPM, CO) (see Fig. 3). (Of note, measurement of mean amplitudes centered around either the A-I or N-I negative peaks yielded the same pattern of results.)

An ANOVA of the mean amplitude revealed an effect of condition $\left(F_{2,48}=6.26, p<0.004\right)$, and a condition $x$ ROI interaction $\left(\mathrm{F}_{8,192}=2.93, \mathrm{p}<0.03\right)$. The effect of condition was due to more negative-going ERPs under both the Attend and Neutral conditions than under the Ignore condition, with no difference between the Attend and Neutral conditions $(A=N<I$; Attend equal to Neutral ( $p>0.4)$; Attend more negative than Ignore $(p<0.005)$; Neutral more negative than Ignore $(\mathrm{p}<0.02)$ ). This resulted in a large negativity $(\mathrm{SN})$ for the Attend - Ignore and Neutral - Ignore difference waves (see Fig. 6). The interaction between condition and ROI was present because the largest difference between conditions was found at the occipito-temporal ROIs, LOT and ROT.

\section{Discussion}

The major purpose of this investigation was to use a color-neutral attention condition to help determine whether modulations of electrophysiological activity associated with attending to color reflect increased activity under an Attend condition, reduced activity under an Ignore condition, or both. The use of physically identical stimuli under all conditions kept bottomup sensory information constant, permitting us to focus on top-down control activity and their modulatory effects (Rutman et al., 2010). Comparing differences in response to nontarget (standard) stimuli under color attend, ignore, and neutral conditions allowed for the isolation of color selective processing.

The prolonged RTs in the SA task provide behavioral evidence that having to take color into account in order to perform a task increased its difficulty. The behavioral results are 
consistent with the electrophysiological data suggesting that the task in which color was relevant elicited additional cognitive operations, which presumably required more time to execute.

Consistent with numerous reports in the literature, neither the P1 nor N1 component yielded an effect of attentional condition, suggesting no overall modulation of these sensory-evoked components by attention to color. The N1 (N170) was not larger over the left than right occipital-temporal region. Thus, we found no clear ERP support for the notion that single letters in our study elicited a word N170, which, if present, would have provided evidence that letter stimuli were being processed like words in a rapid, automatic fashion. However, the design of this study did not offer an opportunity to more definitively examine this issue, which would involve comparing the N170 to letter vs. non-letter stimuli.

As in previous studies, electrophysiological activity was modulated under Attend relative to Ignore conditions during the temporal intervals of the anterior SP and posterior SN. These ERP components have been interpreted as modulations of electrophysiological activity in response to attention to specified features. As noted in the Introduction, there is evidence that the posterior SN represents the enhancement of sensory-perceptual processing of relevant stimulus features compared to irrelevant ones (Harter and Aine, 1984; Hillyard et al., 1998a; Kopp et al., 2007), and the anterior SP may reflect the motivational salience of a stimulus based on task-relevant features (Luck and Hillyard, 1994; Potts and Tucker, 2001; Riis et al., 2009). The generation of SP and SN components provide strong support for early color selection. These components peaked around $180 \mathrm{~ms}$ and $275 \mathrm{~ms}$ respectively, which is hundreds of milliseconds earlier than the P3b to targets (peaking 550 ms in this study), which is considered a marker of late selection (Anllo-Vento and Hillyard, 1996; Hillyard and Munte, 1984; Looren et al., 1988).

For both the anterior SP and the posterior SN, no differences in amplitude were found between the Attend and Neutral conditions, suggesting that neural activity is not specifically enhanced under the Attend condition. The results for the SP require further comment. Examining the mean amplitude during the this component's interval (130-230 ms) yielded no difference in the size of response under the Attend and Neutral conditions, and not even a suggestion of a trend. However, positive activity was sustained longer under Attend than Neutral, which was confirmed by analyzing the mean amplitude between 200-230 ms. Whether this activity is part of the SP component or represents another cognitive operation remains to be determined. Our provisional interpretation is that during the entire interval of the SP, the magnitude of the response does not differ between the Attend and Neutral conditions, with the neural response being larger under Neutral in the early phase, and larger under Attend in the late phase. However, we acknowledge that an alternative interpretation would suggest that there is enhancement of activity under the Attend condition during the final portion of the SP. ${ }^{5}$ This ambiguity is not present for the SN.

\footnotetext{
${ }^{5}$ We acknowledge that alternative inferences might be drawn if a different temporal interval was chosen to compute the SP. For example, if the SP were measured as the mean value between 170-240 ms (and not 130-230 ms), the value of A-I would be similar to that of N-I (see Fig. 5a). The choice of the temporal window in which to measure any ERP component includes some degree of subjectivity. However, we tried to limit the arbitrary nature of the temporal window for SP. It was derived by first determining local positive peak latency for the A-I and N-I difference waves over a large temporal interval, 100-275 ms, and then measuring mean amplitude of the $100 \mathrm{~ms}$ interval centered at the mean peak latency. Using a $100 \mathrm{~ms}$ interval to measure the mean amplitude of endogenous waves like the SP and SN is in range with many other reports in the literature (e.g., Hillyard and Munte, 1984; Kopp et al., 2007; Schoenfeld et al., 2007; Talsma et al., 2006).

To confirm that the interval derived (130-230 ms) was appropriate, we also examined the grand average ERPs of the anterior P2. The initial phase of the anterior P2 crossed the $\mathrm{x}$-axis at $\sim 130 \mathrm{~ms}$, peaked at $\sim 175 \mathrm{~ms}$, and returned to the $\mathrm{x}$-axis between $\sim 200-230 \mathrm{~ms}$ (see Fig. 4a), which approximates the temporal interval that was used in our measurement of the SP. As noted in the text, we also validated our findings by measuring the local peak amplitude of the anterior P2 between 100-275 ms, which circumvents the issue about choosing an arbitrary interval in which to measure the mean amplitude.
} 
Both the anterior SP and posterior SN exhibited reduced responses under the Ignore relative to the Neutral condition. We interpret the reduction in neural activity under the Ignore condition as a reflection of top-down suppression of the processing of the to-be-ignored feature. One potential challenge to this account relates to the endogenous nature of the SP and SN components. Unlike our study, most research that has taken advantage of a neutral condition (e.g., Gazzaley et al., 2008; Luck et al., 1994; Mangun and Hillyard, 1990) has focused on the modulation of sensory-evoked, exogenous components like the posterior P1 and posterior N1. Sensory-evoked components represent relatively automatic responses to stimuli that are elicited regardless of task demand or direction of attention. Under such circumstances, a smaller response under ignore than neutral would unequivocally imply topdown suppression of this obligatory response. In contrast, the SP and SN represent endogenous components, which by definition are task-dependent, require attention, and are not obligatory. One could argue that the absence of a response under the Ignore condition is simply due to the fact that endogenous activity is not elicited in this context. However, based on the logic presented in the Introduction, we believe that the suppression account provides a more adequate explanation of the findings for the SP and SN components in this study. As was suggested, under the SA task, selective attention to specified features is carried out by modulation (i.e., enhancement or suppression) of operations elicited under the NA task. This modulation accounts for the difference in activity between the Attend and Ignore conditions. In the absence of clearly enhanced neural activity associated with selective attention ( $\mathrm{A}=$ $\mathrm{N})$, the difference between the Attend and Ignore conditions must therefore be due to the suppression of activity under the Ignore condition. Moreover, as suggested by Zanto and colleagues (2010a), when subjects are presented with a sequence of stimuli that include events to be attended or ignored, the default response may be to attend. In this context, ignoring stimuli may require additional processing associated with suppression.

One strategy for further investigating whether a smaller electrophysiological response under the Ignore condition represents the suppression of neural activity or simply the absence of eliciting a response is to examine the pattern of activity in other clinical groups. For example, our preliminary analysis of a group of older adults (65-79 years old) suggests that unlike young adults, whose pattern for the Selection Positivity is A = N > I, the pattern of response in older adults is $\mathrm{A}>\mathrm{N}=\mathrm{I}$. In keeping with the inhibitory-deficit hypothesis of aging (Dempster, 1992), this finding would indicate that unlike young adults, older adults may fail to inhibit neural activity under the Ignore condition (Gazzaley et al., 2008; Zanto et al., 2010a).

Another potential challenge to our formulation involves alternative interpretations of the functional significance of the SP and SN. For example, we have adopted the traditional view that the SN reflects an early selection mechanism, involving the modulation of neural activity of color sensitive neurons in visual association cortex that augments sensoryperceptual processing of attend relative to ignore features. An alternative account of the SN is that it represents a late strategic process, such as updating memory traces, or scanning memory to determine if a stimulus contains target characteristics (Kenemans et al., 1995; Zanto et al., 2010b). If the SN represented a late selection mechanism like memory scanning, the prevention of such processing in response to stimuli in the ignore color would seem to require that these stimuli had already been identified on the basis of their color. However, there is no evidence from the current data set to suggest that the brain differentially responds to color as a function of attentional demands earlier than the generation of the SP and SN. ${ }^{6}$ Although the current study cannot resolve questions surrounding the functional significance of the $\mathrm{SN}$, we would emphasize that the critical issue here is that neural processing indexed by this component is distinctly different in response to stimuli under the color Ignore condition than under the color Attend or Neutral conditions. When the brain is presented with a stimulus in the to-be-ignored color, this neural activity is 
minimal. We have offered arguments above for why we believe that this result is a reflection of a top-down inhibitory process.

Our results differ in a several ways from those reported in studies by Gazzaley and colleagues $(2005 \mathrm{a}$; 2005b; 2008). For example, in contrast to our findings, they report attentional modulation of the P1 amplitude and the N1 latency. It seems likely that differences in experimental design between our study and those of Gazzaley and colleagues help account for the discrepant findings. For example, the kinds of visual stimuli presented were very different (e.g., faces/scenes in the Gazzaley et al. studies vs. red/blue letters in our studies). Even in Gazzaley et al. studies, attention to scenes did not modulate the P1 and N1 components. In addition, subjects in their studies have been asked to direct attention to one of two kinds of stimuli, whereas subjects in the current study were required to attend to a particular exemplar of a feature while ignoring another. The investigations by Gazzaley et al. examined neural activity in response to working memory encoding in which stimuli changed on each trial, whereas our study involved target detection in which the attend color and letter targets remained constant. ${ }^{7}$

In contrast to our study, Gazzalley et al. found evidence not only of suppressed neural activity under the ignore relative to the neutral condition, but also robustly enhanced neural activity under the attend relative to the neutral condition. Substantial differences in the kind of neutral condition employed may have influenced the pattern of results. Similar to studies by Luck et al. (1994) and Mangun and Hillyard (1990), our Neutral condition required a subject to actively attend to the task, but not exercise selective attention operations. By contrast, the studies by Gazzaley and colleagues employed a neutral condition that involved passive viewing of visual stimuli, which may have differed from the attend condition not only in terms of directed attention to a specified feature, but also the general level of attentional focus, effort, and motivation. Their finding of enhanced neural activity under the attend relative to the passive condition may reflect the impact of these other processes.

Consistent with the idea that a range of factors could influence the ways in which neural responses vary across attentional conditions are the results of investigations by Müller and colleagues (Andersen and Müller, 2010; Müller et al., 1998). Their studies of selective attention to color using steady state visual evoked potentials (SSVEPs) and the pre-cue baseline as the neutral condition suggest that whether one finds increased or decreased neural activity relative to baseline depends on the nature of the cue; a spatial attention cue was associated with enhancement of activity under attend (Müller et al., 1998), whereas as a color cue was associated with both enhancement under attend and suppression under ignore (Andersen and Müller, 2010). These differing results in the literature reinforce the notion that attention involves a complex set of operations that are sensitive to a variety of factors, which can affect measurements suggestive of enhancement and suppression. The interpretation of our findings applies most directly to processes indexed by the SN and SP under a selective attention/working memory task in which stimuli are sequentially presented

\footnotetext{
${ }^{6}$ We explored the possibility that the SN reflects a memory scanning process for target characteristics by comparing the SN in response to standard stimuli to the $\mathrm{SN}$ in response to target stimuli. If the $\mathrm{SN}$ is a late selection process that utilizes information about both color and letter forms, one might expect the SN in response to target letters to be larger than in response to standard letters. First, we measured the peak negative latency of the difference waves in the $200-350 \mathrm{~ms}$ interval (A-I and N-I) for targets vs. standards and determined that there was no effect of stimulus type $(p>0.67)$ and no stimulus $x$ difference wave interaction $(p>0.17)$. Next, we examined the mean amplitude 225-325 ms for standards vs. targets at posterior ROIs across the 3 conditions. There was no effect of stimulus type $(p>0.11)$ and no stimulus type $x$ condition interaction $(p>0.26)$. These results indicate that there were no differences between standard stimuli and target stimuli in the amplitude and latency of the SN.

${ }^{7}$ It also remains to be determined what experimental conditions are associated with the modulation of early sensory evoked potentials (e.g., P1 or N1) in response to attention to color, as was reported by Zanto et al. (2010b; Zanto and Gazzaley, 2009), but was absent in the current study and many others in the literature. Possibilities include 1) changing stimulus set on each working memory encoding trial, 2) directing attention to one of two feature dimensions (e.g., color vs. motion), and 3) increasing attentional demands (e.g., simultaneously rather than sequentially presenting attend and ignore colors (Zhang and Luck, 2009).
} 
at a central location, the task-relevant color remains the same throughout the experiment, and the neutral condition is an active one that includes all requirements of the selective attention condition except for color selectivity.

In summary, despite over 25 years of productive research on ERP markers of early selection, the anterior Selection Positivity and the posterior Selection Negativity in response to color, there has been limited investigation of whether the observed differences in neural activity between the attend and ignore conditions reflect enhancement or inhibition. The inclusion of a color-neutral attention task provided the experimental context in which to determine that the SP and SN primarily reflect the modulation of neural activity under the ignore, not the attend condition. The results are not consistent with theories about top-down modulation of neural activity that do not include dedicated mechanisms for inhibition (Braver and Barch, 2002). Additional research is necessary to better understand the relationship between these early inhibitory processes and subsequent cognitive operations, and to track the ways in which disruption of early stages may impact later processing (Gazzaley et al., 2008; Zanto and Gazzaley, 2009).

\section{Acknowledgments}

This research was funded in part by NIA grant R01 AGO17935 and by generous support from D. Wimberly and S. Muss. The authors would like to thank Katie Gartner and Marissa Cabrera for their excellent administrative assistance.

\section{References}

1. American Psychiatric Association. Diagnostic and Statistical Manual of Mental Disorders. 4. Washington, D.C: American Psychiatric Association; 1994.

2. Andersen SK, Müller MM. Behavioral performance follows the time course of neural facilitation and suppression during cued shifts of feature-selective attention. Proc Natl Acad Sci U S A. 2010; 107:13878-13882. [PubMed: 20643918]

3. Anllo-Vento L, Hillyard SA. Selective attention to the color and direction of moving stimuli: electrophysiological correlates of hierarchical feature selection. Percept Psychophys. 1996; 58:191206. [PubMed: 8838164]

4. Awh E, Jonides J. Overlapping mechanisms of attention and spatial working memory. Trends Cogn Sci. 2001; 5:119-126. [PubMed: 11239812]

5. Beck, AT.; Steer, RA. Beck Depression Inventory: Manual. San Antonio, TX: The Psychological Corporation; 1987.

6. Besner D, Stolz JA, Boutilier C. The stroop effect and the myth of automaticity. Psychon Bull Rev. 1997; 4:221-225. [PubMed: 21331828]

7. Braver TS, Barch DM. A theory of cognitive control, aging cognition, and neuromodulation. Neurosci Biobehav Rev. 2002; 26:809-817. [PubMed: 12470692]

8. Brown TL, Joneleit K, Robinson CS, Brown CR. Automaticity in reading and the Stroop task: testing the limits of involuntary word processing. Am J Psychol. 2002; 115:515-543. [PubMed: 12516527]

9. Czigler I. Age, color processing and meaningfulness: an event-related potential study. Int J Psychophysiol. 1996; 22:25-34. [PubMed: 8799765]

10. Delorme A, Makeig S. EEGLAB: an open source toolbox for analysis of single-trial EEG dynamics including independent component analysis. J Neurosci Methods. 2004; 134:9-21. [PubMed: 15102499]

11. Dempster FN. The rise and fall of the inhibitory mechanism: Toward a unified theory of cognitive development and aging. Developmental Review. 1992; 12:45-75.

12. Desimone R, Duncan J. Neural mechanisms of selective visual attention. Annu Rev Neurosci. 1995; 18:193-222. [PubMed: 7605061] 
13. Eimer M. An event-related potential (ERP) study of transient and sustained visual attention to color and form. Biol Psychol. 1997; 44:143-160. [PubMed: 9043651]

14. Gazzaley A, Clapp W, Kelley J, McEvoy K, Knight RT, D'Esposito M. Age-related top-down suppression deficit in the early stages of cortical visual memory processing. Proc Natl Acad Sci U S A. 2008; 105:13122-13126. [PubMed: 18765818]

15. Gazzaley A, Cooney JW, McEvoy K, Knight RT, D'Esposito M. Top-down enhancement and suppression of the magnitude and speed of neural activity. J Cogn Neurosci. 2005a; 17:507-517. [PubMed: 15814009]

16. Gazzaley A, Cooney JW, Rissman J, D'Esposito M. Top-down suppression deficit underlies working memory impairment in normal aging. Nat Neurosci. 2005b; 8:1298-1300. [PubMed: 16158065]

17. Harter, RM.; Aine, CJ. Brain Mechanisms of Visual Selective Attention. In: Parasuraman, R.; Davies, DR., editors. Varieties of Attention. Academic Press Inc; 1984. p. 293-321.

18. Hillyard SA, Anllo-Vento L. Event-related brain potentials in the study of visual selective attention. Proc Natl Acad Sci U S A. 1998; 95:781-787. [PubMed: 9448241]

19. Hillyard SA, Munte TF. Selective attention to color and location: an analysis with event-related brain potentials. Percept Psychophys. 1984; 36:185-198. [PubMed: 6514528]

20. Hillyard SA, Teder-Salejarvi WA, Munte TF. Temporal dynamics of early perceptual processing. Curr Opin Neurobiol. 1998a; 8:202-210. [PubMed: 9635203]

21. Hillyard SA, Vogel EK, Luck SJ. Sensory gain control (amplification) as a mechanism of selective attention: electrophysiological and neuroimaging evidence. Philos Trans R Soc Lond B Biol Sci. 1998b; 353:1257-1270. [PubMed: 9770220]

22. Kenemans JL, Smulders FT, Kok A. Selective processing of two-dimensional visual stimuli in young and old subjects: electrophysiological analysis. Psychophysiology. 1995; 32:108-120. [PubMed: 7630975]

23. Kopp B, Tabeling S, Moschner C, Wessel K. Temporal dynamics of selective attention and conflict resolution during cross-dimensional Go-NoGo decisions. BMC Neurosci. 2007; 8:68-83. [PubMed: 17705856]

24. Lavie N, Hirst A, de Fockert JW, Viding E. Load theory of selective attention and cognitive control. J Exp Psychol Gen. 2004; 133:339-354. [PubMed: 15355143]

25. Looren, dJ; Kok, A.; van Rooy, JC. Early and late selection in young and old adults: an eventrelated potential study. Psychophysiology. 1988; 25:657-671. [PubMed: 3241853]

26. Luck SJ, Hillyard SA, Mouloua M, Woldorff MG, Clark VP, Hawkins HL. Effects of spatial cuing on luminance detectability: psychophysical and electrophysiological evidence for early selection. J Exp Psychol Hum Percept Perform. 1994; 20:887-904. [PubMed: 8083642]

27. Luck S, Hillyard SA. Electrophysiological correlates of feature analysis during visual search. Psychophysiology. 1994; 31:291-308. [PubMed: 8008793]

28. MacLeod CM. Half a century of research on the Stroop effect: an integrative review. Psychol Bull. 1991; 109:163-203. [PubMed: 2034749]

29. Mangun GR, Hillyard SA. Allocation of visual attention to spatial locations: tradeoff functions for event-related brain potentials and detection performance. Percept Psychophys. 1990; 47:532-550. [PubMed: 2367174]

30. Martin-Loeches M, Hinojosa JA, Rubia FJ. Insights from event-related potentials into the temporal and hierarchical organization of the ventral and dorsal streams of the visual system in selective attention. Psychophysiology. 1999; 36:721-736. [PubMed: 10554586]

31. Maurer U, Rossion B, McCandliss BD. Category specificity in early perception: face and word n170 responses differ in both lateralization and habituation properties. Front Hum Neurosci. 2008; 2:18. [PubMed: 19129939]

32. Müller MM, Teder-Salejarvi W, Hillyard SA. The time course of cortical facilitation during cued shifts of spatial attention. Nat Neurosci. 1998; 1:631-634. [PubMed: 10196572]

33. Potts GF, Tucker DM. Frontal evaluation and posterior representation in target detection. Brain Res Cogn Brain Res. 2001; 11:147-156. [PubMed: 11240117] 
34. Riis JL, Chong H, McGinnnis S, Tarbi E, Sun X, Holcomb PJ, Rentz DM, Daffner KR. Agerelated changes in early novelty processing as measured by ERPs. Biol Psychol. 2009; 82:33-44. [PubMed: 19463888]

35. Rossion B, Joyce CA, Cottrell GW, Tarr MJ. Early lateralization and orientation tuning for face, word, and object processing in the visual cortex. NeuroImage. 2003; 20:1609-1624. [PubMed: 14642472]

36. Rutman AM, Clapp WC, Chadick JZ, Gazzaley A. Early top-down control of visual processing predicts working memory performance. J Cogn Neurosci. 2010; 22:1224-1234. [PubMed: 19413473]

37. Ryan J, Paolo A. A screening procedure for estimating premorbid intelligence in the elderly. Clin Neuropsychol. 1992; 6:53-62.

38. Sawaki R, Katayama J. Top-down directed attention to stimulus features and attentional allocation to bottom-up deviations. J Vis. 2008; 8:4-8. [PubMed: 19146288]

39. Schoenfeld MA, Hopf JM, Martinez A, Mai HM, Sattler C, Gasde A, Heinze HJ, Hillyard SA. Spatio-temporal analysis of feature-based attention. Cereb Cortex. 2007; 17:2468-2477. [PubMed: 17204821]

40. Talsma D, Kok A, Ridderinkhof KR. Selective attention to spatial and non-spatial visual stimuli is affected differentially by age: effects on event-related brain potentials and performance data. Int $\mathrm{J}$ Psychophysiol. 2006; 62:249-261. [PubMed: 16806547]

41. Turkeltaub PE, Flowers DL, Lyon LG, Eden GF. Development of ventral stream representations for single letters. Ann N Y Acad Sci. 2008; 1145:13-29. [PubMed: 19076386]

42. Van Der Stelt O, Kok A, Smulders FT, Snel J, Boudewijn GW. Cerebral event-related potentials associated with selective attention to color: developmental changes from childhood to adulthood. Psychophysiology. 1998; 35:227-239. [PubMed: 9564743]

43. Zanto TP, Gazzaley A. Neural suppression of irrelevant information underlies optimal working memory performance. J Neurosci. 2009; 29:3059-3066. [PubMed: 19279242]

44. Zanto TP, Hennigan K, Ostberg M, Clapp WC, Gazzaley A. Predictive knowledge of stimulus relevance does not influence top-down suppression of irrelevant information in older adults. Cortex. 2010a; 46:564-574. [PubMed: 19744649]

45. Zanto TP, Toy B, Gazzaley A. Delays in neural processing during working memory encoding in normal aging. Neuropsychologia. 2010b; 48:13-25. [PubMed: 19666036]

46. Zhang W, Luck SJ. Feature-based attention modulates feedforward visual processing. Nat Neurosci. 2009; 12:24-25. [PubMed: 19029890] 


\section{Highlights}

- We examine mechanisms underlying selective attention to color using ERPs

- Responses under color-attend, color-ignore, and color-neutral conditions are compared

- No differences between attend and neutral in ERP markers of early selection

- Marked reduction of ERP responses under ignore relative to neutral condition

- Early selection reflects suppression of neural activity under the ignore condition 


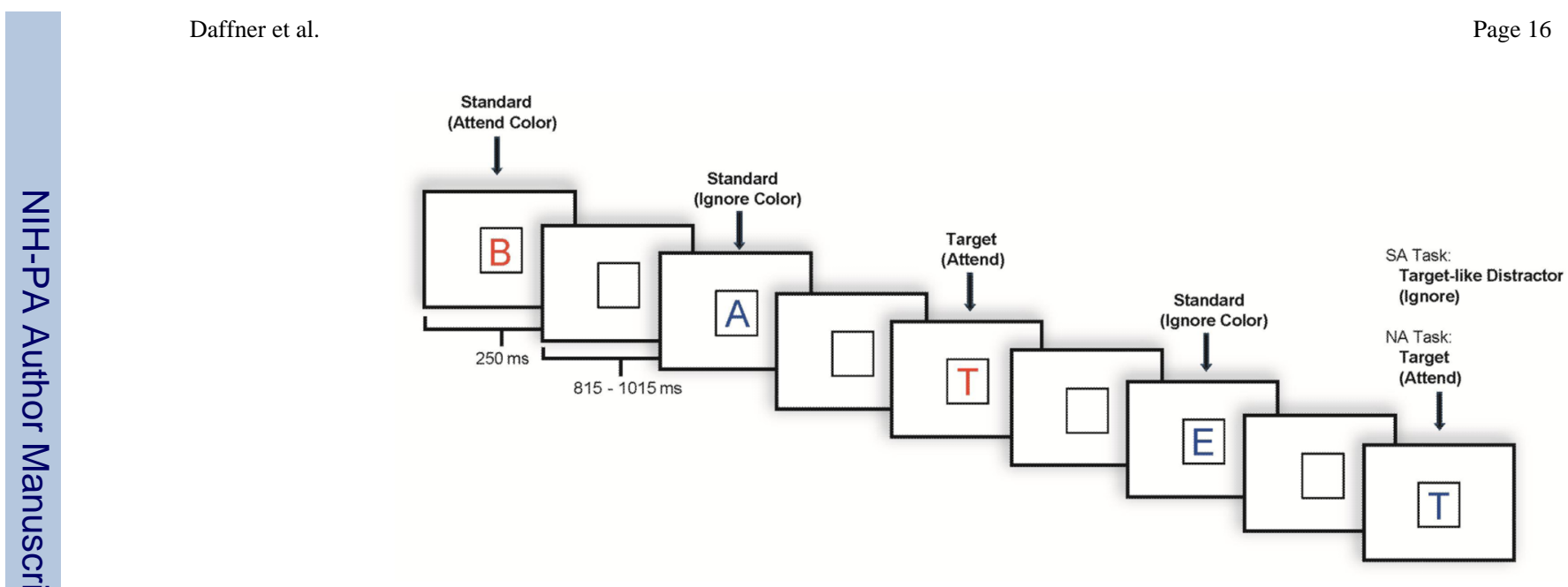

Figure 1.

Illustration of an experimental run. 


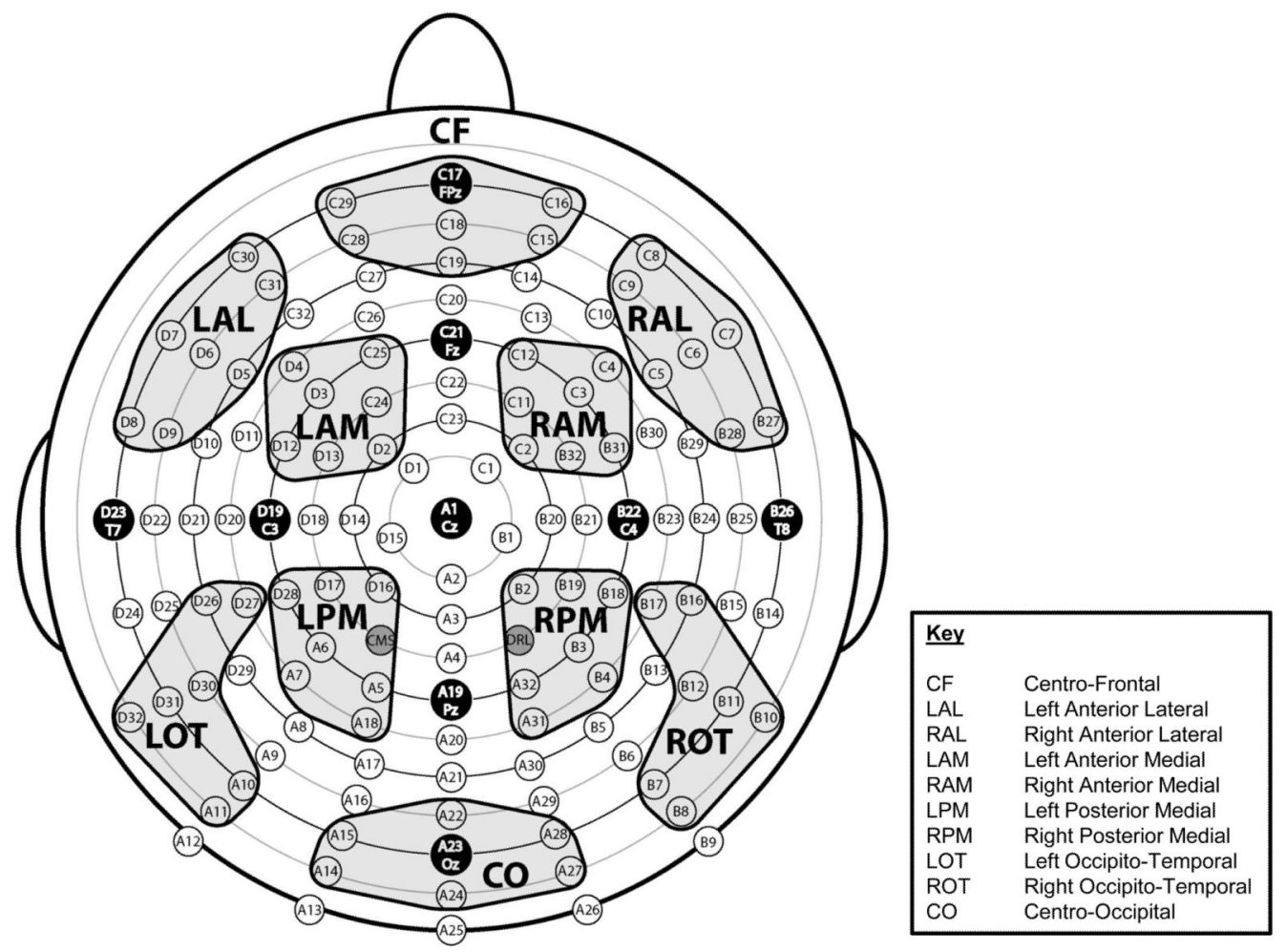

Figure 2.

Montage illustrating the location of 128 electrode sites and the 10 designated regions of interest (ROIs). 


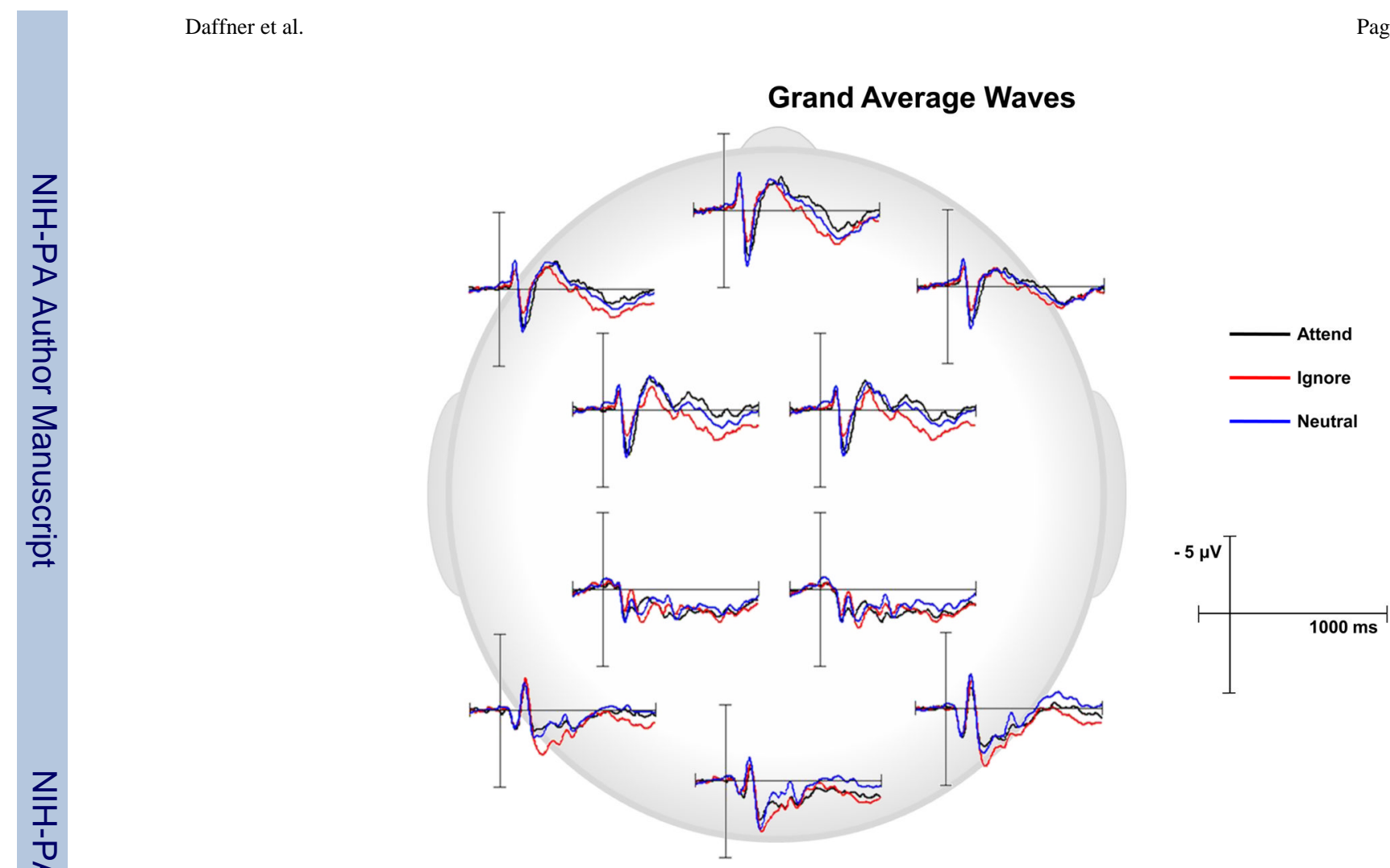

Figure 3.

Grand average ERP responses to standard stimuli under Attend, Ignore, and Neutral conditions at all 10 ROIs. 


\section{Grand Average Waves}

a) Anterior ROI: CF
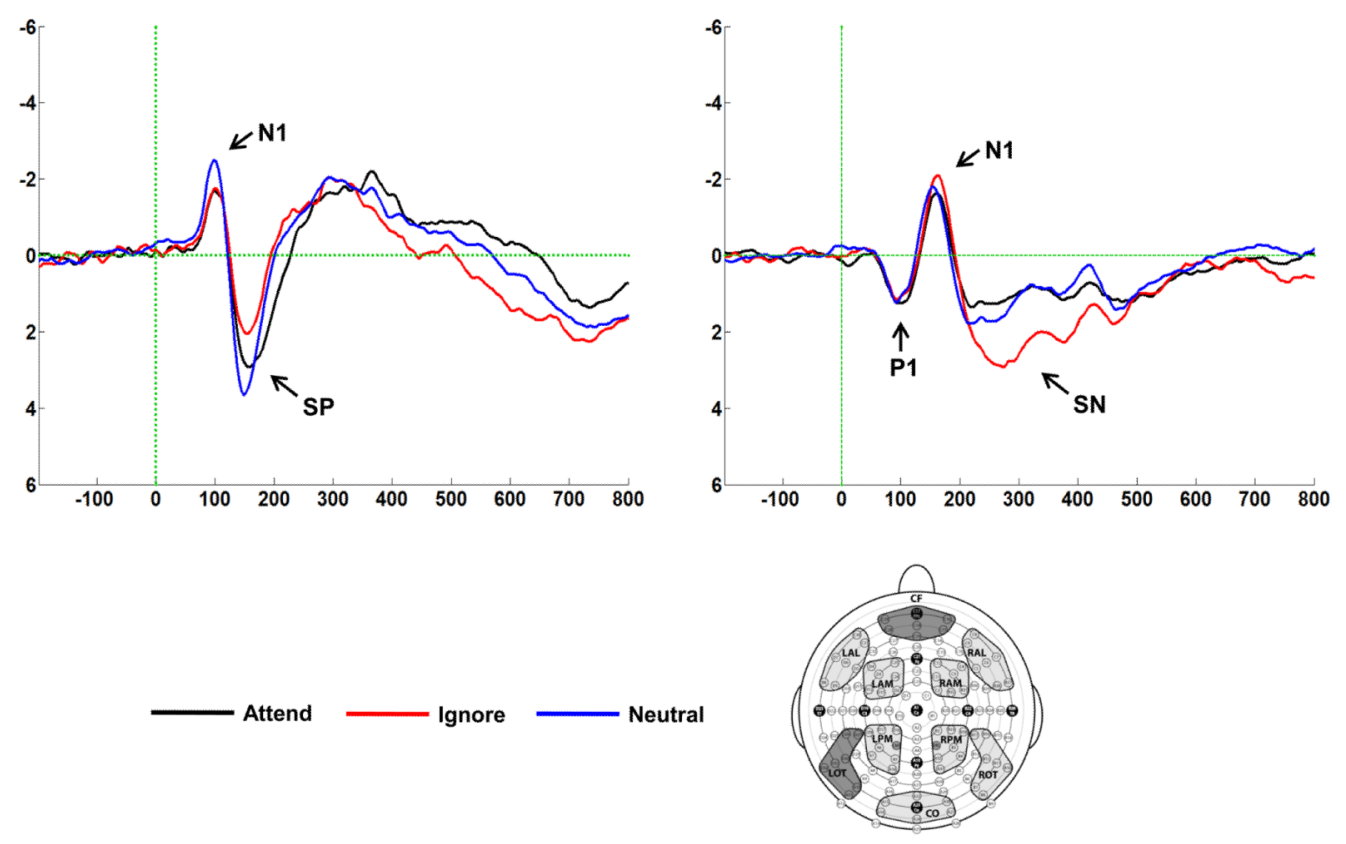

Figure 4.

Grand average ERPs at the a) centro-frontal (CF) ROI, illustrating the anterior N1 component and the Selection Positivity (SP), and b) left occipito-temporal (LOT) ROI, illustrating the P1 component, N1 component, and Selection Negativity (SN). 


\section{Selection Positivity (SP)}

\section{a) Difference Waves}
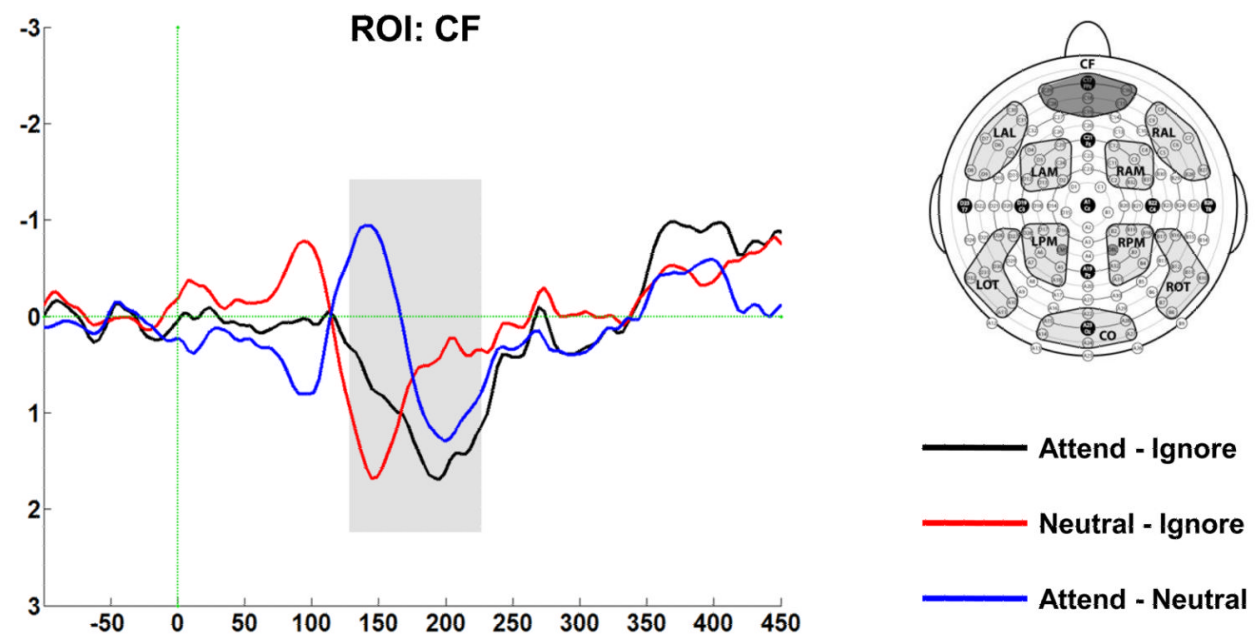

b) Topographical Maps of Difference Waves

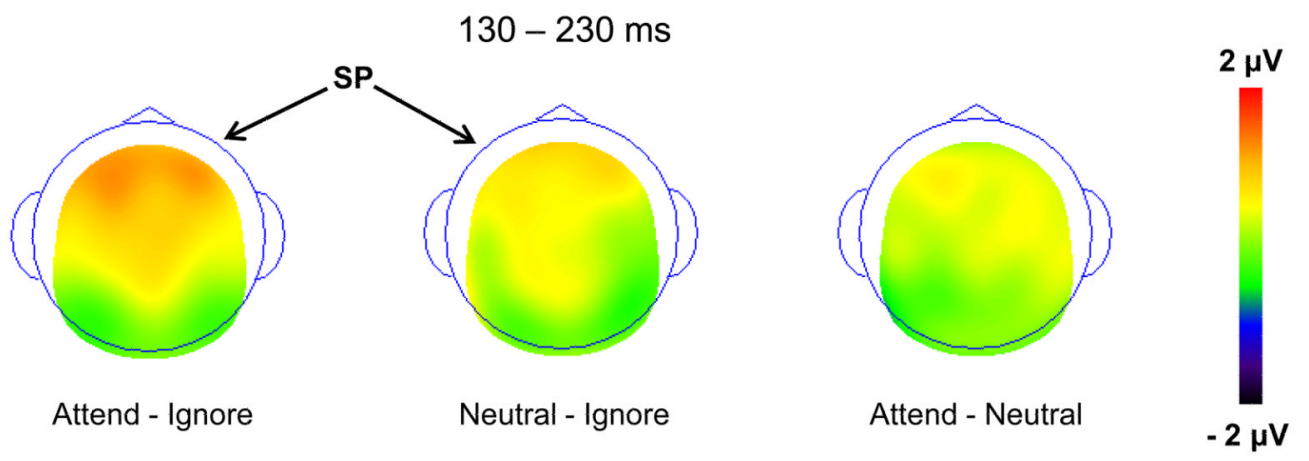

Figure 5.

a) Illustration of the differences waves (Attend - Ignore, Neutral - Ignore, and Attend Neutral at the centro-frontal (CF) ROI. Highlighted area indicates the temporal interval of the Selection Positivity (SP); b) Surface potential maps of differences waves under Attend Ignore, Neutral - Ignore, and Attend - Neutral conditions for the SP interval (130-230 ms). Arrows illustrate the presence of the SP. 


\section{Selection Negativity (SN) \\ a) Difference Waves}
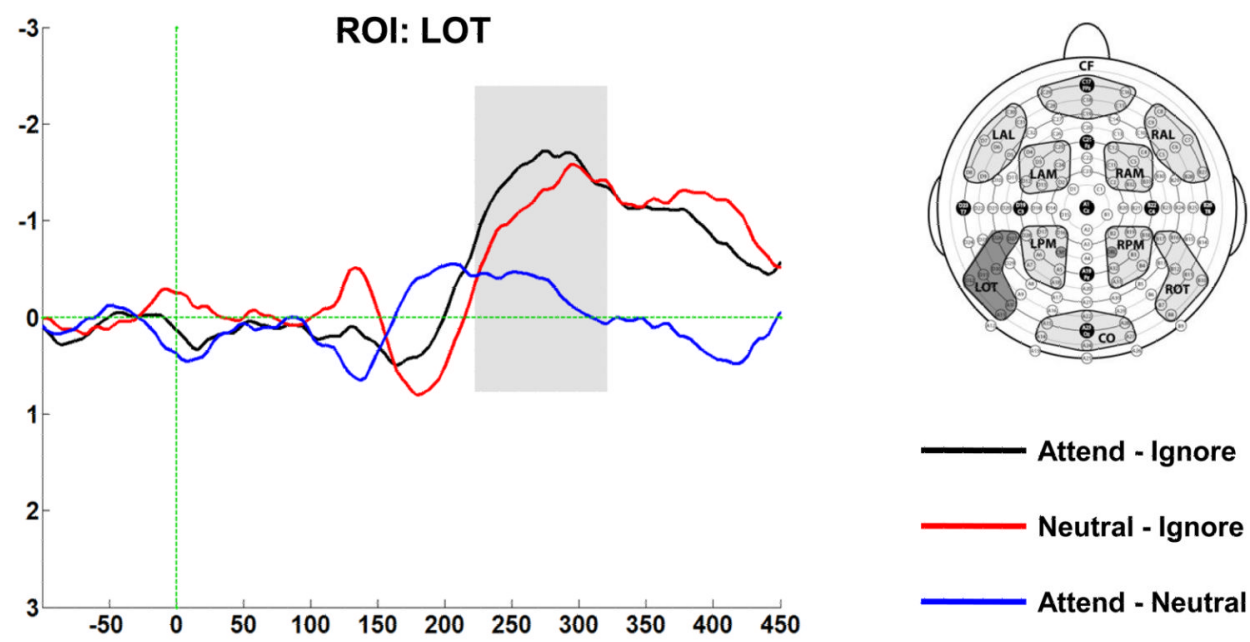

Neutral - Ignore

Attend - Neutral

\section{b) Topographical Maps of Difference Waves}

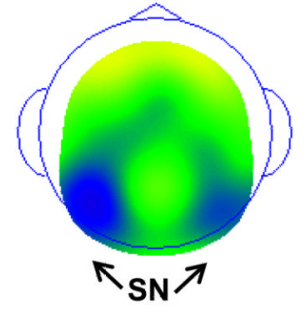

Attend - Ignore

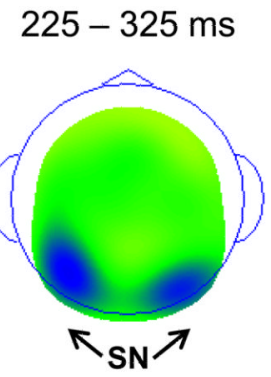

Neutral - Ignore

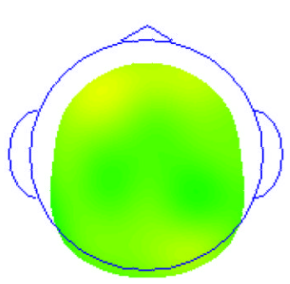

Attend - Neutral

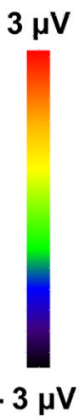

Figure 6.

a) Illustration of the differences waves (Attend - Ignore, Neutral - Ignore, and Attend Neutral at the left occipito-temporal (LOT) ROI. Highlighted area indicates the temporal interval of the Selection Negativity (SN); b) Surface potential maps of difference waves under Attend - Ignore, Neutral - Ignore, and Attend - Neutral conditions for the SN interval (225-325 ms). Arrows illustrate the presence of the SN. 